\title{
Integrative Levels of Granularity
}

\author{
Yiyu Yao \\ Department of Computer Science, University of Regina, Regina, Saskatchewan, Canada S4S \\ 0A2, Email: yyao@cs.uregina.ca
}

\begin{abstract}
In their book, Granular Computing: An Introduction, Bargiela and Pedrycz present a view that granular computing is an emerging conceptual and computing paradigm of information processing. A central notion is an information-processing pyramid with multiple levels. Different levels involve different types of processing. The lowest level concerns numeric processing, the intermediate level concerns larger information granules, and the highest level concerns symbol-based processing. This chapter examines the notion of integrative levels of granularity as a basis of granular computing. The notion of levels had been studied extensively in different branches of sciences and different fields of computer sciences. By extracting a set of common features and principles of integrative levels of granularity, the triarchic theory of granular computing is developed.
\end{abstract}

\section{Introduction}

Granular computing is a multi-disciplinary and cross-disciplinary study (see, for examle, Bargiela and Pedrycz [3, 5], Inuiguchi et al. [20], Keet [21,22], Nguyen et al. [34], Lin et al. [27], Pawlak [38], Pedrycz et al. [39], Yao, JT [53], Yao [54], Zadeh [63], Zhang and Zhang [64]), concerning problem solving and information processing with multiple levels of granularity [55-59]. Granular computing may be viewed as human-inspired paradigms of computing and information processing, as well as their applications in the design and implementation of intelligent information systems $[5,61]$.

In their book, Granular Computing: An Introduction, Bargiela and Pedrycz [3] promote granular computing as an emerging conceptual and computing paradigm of information processing. The working principles of granular computing are explained based on an information-processing pyramid with multiple levels. Different levels involve different types of processing. The lowest level concerns numeric processing, the intermediate level concerns larger information granules, and the highest level concerns symbol-based processing. From this conceptual framework, we can identify two important notions, namely, granules and granular 
structures. Granules are elements and units that build up levels and granular structures are levels partially ordered based on their granularity. The formal representation of a granular structure as a multilevel hierarchical structure is based on further results from systems science, artificial intelligence and computer programming [57-59].

This chapter covers two aspects of granular computing. In Sections 2 to 4, we show that the notion of levels plays a fundamental role in many branches of sciences. A survey on many different interpretations and uses suggests that the concept of integrative levels of granularity may serve as a basis of granular computing. In Section 5, we briefly discuss the triarchic theory of granular computing that is centered around granular structures.

\section{Integrative Levels}

In this section, we argue that levels and associated multilevel hierarchical structures are common words of languages used in a wide spectrum of disciplines. A few important features of levels are examined.

\subsection{Universality of levels}

The notions of levels and associated multilevel hierarchical structures are perhaps some of the most fundamental concepts and tools that we use to describe, represent, analyze and understand ourselves, reality and our relations to reality $[1,8$, $11,35,41,46,57,62]$. As pointed out by Conger [8], the interpretations of differences of level range from the literal meanings to the various metaphorical meanings. The term "levels" seems to be a universal concept that has been widely used in philosophy and virtually all branches of natural and social sciences.

A (metaphysical) level, as defined by Conger [8], is "a class of structures or processes which are distinguishable from others as being either higher or lower." The terms "higher" and "lower" may denote various spatial, valuational, logical and developmental differences. Independent of any particular interpretation, such a higher-lower relation enables us to order levels, and hence to produce a multilevel hierarchical structure called a hierarchy.

In June 2008, we performed Google searches using a dozen phrases involving "levels." Table 1 summarizes the results about the number of hits of different phrases. Several observations can be made from a closer examination of various usages of levels and hierarchical structures.

Firstly, the notion of "levels" seems to be universally applicable to many different disciplines. The numbers of hits of various phrases containing "levels" range from several thousands to a few millions. Levels and hierarchical structures 
are used in virtually every branch of science and our daily life. Secondly, there seems to be a common understanding of levels, although slightly different interpretations exist in different fields. Levels may have either an objective or a subjective interpretation. The former reflects the intrinsic nature of reality; the latter reflects our cognitive understanding of reality. Levels are used to describe, organize and interpret things for the purposes of simplicity and clarity. Levels are sometimes used to denote a particular position on a scale, as reflected by levels of skill and levels of intelligence. Thirdly, levels are associated with the dual properties of separation and integration, and hence the term "integrative levels" and "integrated levels" are widely used. Levels generally imply a separation of things, with each level focusing on a particular aspect. Levels can be ordered partially to form a hierarchical or nested structure. That is, many levels can be integrated to form a whole. A level can be further divided into sub-levels and many levels can be combined into one level, depending on our point of observation. One needs to study a level in the context of other levels. Fourthly, in theory there may be an arbitrary large number of many levels. However, most common uses of levels normally are within ten. Table 2 shows the numbers of hits on searching the numbers of levels commonly used. It can be seen that the most used numbers of levels are between two to four, with a peak at three. As the number of levels increases, the number of hits decreases. In other words, our understanding of reality is typically at a few levels, instead of a large number of levels.

The numbers of hits in Tables 1 and 2 should be read qualitatively. For example, the " 2 " in the phrase " 2 levels of" may not actually mean that two levels are in fact used. Nevertheless, the observations from the tables are valid at a qualitative level. In particular, the trends obtained from the tables may be correct. For example, the phrase "levels of abstraction" is used more frequently than "the level of description." People prefer a simple two- or three-level structure to other, more complex structures with many more levels. The results of Tables 1 and 2 perhaps deserve further attention and analysis, as they may enhance our understanding of granular computing where the notion of levels is of fundamental importance. In the next two subsections, we will examine in detail two aspects of the notion of levels.

A final note is that the term layers has also been widely used in place of levels. In fact, some authors use them interchangeably [41]. Like multilevel approaches, multilayer methodologies have been extensively studied. For example, a Google search of "multilevel" produces about 4.6 million hits; a search of "multilayer" produces about 4.4 million hits (searches done in November, 2008). In some sense, the term layers suggests a kind of total, linear ordering, namely, one layer on top of another. For levels, we only require a partial ordering. Studies on layers further demonstrate the universality of levels, as both of them roughly represent the same thing. The study of granular computing can also draw results from studies of layer-based approaches and multilayer methodologies. In some situations, the term layers may be intuitively more appealing. 
Table 1 Google search results of various uses of levels

\begin{tabular}{|l|r|}
\hline \multicolumn{1}{|c|}{ Phrase } & \multicolumn{1}{|c|}{ Hits } \\
\hline "levels of ability" & $1,300,000$ \\
\hline "levels of abstraction" & $1,030,00$ \\
\hline "levels of analysis" & 684,000 \\
\hline "levels of business" & 163,000 \\
\hline "levels of complexity" & $2,130,000$ \\
\hline "levels of comprehension" & 142,000 \\
\hline "levels of cognition" & 35,100 \\
\hline "levels of consciousness" & 216,000 \\
\hline "levels of control" & 222,000 \\
\hline "levels of description" & 86,100 \\
\hline "levels of detail" & 333,000 \\
\hline "levels of discovery" & $2,710,000$ \\
\hline "levels of evidence" & 113,000 \\
\hline "levels of experience" & 309,000 \\
\hline "levels of function" & 983,000 \\
\hline "levels of government" & $2,800,000$ \\
\hline "levels of granularity" & 143,000 \\
\hline "levels of intelligence" & 106,000 \\
\hline "levels of interpretation" & 565,000 \\
\hline "levels of intuition" & 14,500 \\
\hline "levels of knowledge" & 207,000 \\
\hline "levels of measurement" & $1,600,000$ \\
\hline "levels of observation" & 27,300 \\
\hline "levels of organization" & 468,000 \\
\hline "levels of perception" & 39,600 \\
\hline "levels of processing" & 226,000 \\
\hline "levels of reality" & 171,000 \\
\hline "levels of reasoning" & 425,000 \\
\hline "levels of representation" & 932,000 \\
\hline "levels of skill" & 361,000 \\
\hline "levels of strategy" & $1,220,000$ \\
\hline "levels of thinking" & 168,000 \\
\hline "levels of thought" & 32,000 \\
\hline "levels of understanding" & 245,000 \\
\hline
\end{tabular}


Table 2. Google search results of the numbers of levels.

\begin{tabular}{|c|r|c|r|}
\hline Phrase & \multicolumn{1}{|c|}{ Hits } & Phrase & \multicolumn{1}{c|}{ Hits } \\
\hline "two levels of" & $3,510,000$ & "2 levels of" & 636,000 \\
\hline "three levels of" & $4,130,000$ & "3 levels of" & $1,330,000$ \\
\hline "four levels of" & $1,460,000$ & "4 levels of" & 477,000 \\
\hline "five levels of" & 754,000 & "5 levels of" & 557,000 \\
\hline "six levels of" & 284,000 & "6 levels of" & 191,000 \\
\hline "seven levels of" & 195,000 & "7 levels of" & 141,000 \\
\hline "eight levels of" & 122,000 & "8 levels of" & 146,000 \\
\hline "nine levels of" & 40,300 & "9 levels of" & 79,900 \\
\hline "ten levels of" & 60,500 & "10 levels of" & 99,400 \\
\hline
\end{tabular}

\subsection{Objective and subjective views of levels}

Levels and hierarchical structures are used to represent both reality and our perception and conceptualization of reality. Pattee [37] suggests that hierarchical systems may be characterized by the requirement of levels of description and the requirement of levels of structure. The requirement of levels of structure captures the inherent nature of a complex system, and the requirement of levels of description captures our understanding of the complex system. Poli [41] makes a similar distinction between the levels of reality and the levels of description. "The levels of reality have a strictly ontological valence, while those of description have a strictly epistemological one" [41]. A critical question put forward by Young [62], based on several other studies, is that of "whether hierarchies really exist or are simply a fiction of organizational convenience." These studies suggest two extreme views for the interpretation of levels and a hierarchy, dealing with both the objective nature and the subjective nature of a hierarchy [22, 57]. Verdier [50] summarizes the two extremes as "the proponents of a hierarchy that is to be discovered" and "the proponents of an elaboration of a hierarchy by researchers." In other words, the objective view is based on a position that the structural levels of matter are determined by the entirely objective laws of nature. Examples of this view include the levels of organization, the levels of control, and many more. The subjective view focuses on the human subjective multilevel understanding of reality. A hierarchy is formulated and built by the levels of description of our choice, which is based on our understanding through laws of the nature and the results of our observations. Examples of this view include the levels of description, the levels of representation, the levels of analysis, and many others.

At the same time, it may be not so easy to separate reality and our perception and understanding of reality, as described by the two views. It may be argued that we adopt the corresponding levels of description to reflect reality. In other words, our descriptions, in the form of hierarchical structures, merely mirror reality. For 
example, Hawkins [18] proposes that the human brain can be interpreted as a hierarchical structure that stores a model of the hierarchical structure of the real world. The real world's nested structure is mirrored by the nested structure of our cortex. Many of the phrases in Table 1 in fact reflect both the objective and subjective nature of levels.

The objective view on the existence of multilevel hierarchical structures may be explained in an evolutionary framework of complex systems proposed by Simon [47]. It is suggested that a hierarchy emerges almost inevitably through evolutionary processes for reasons of efficiency and stability. Systems can be quickly evolved to hierarchical structures and such structures are stable.

The subjective view on our imposition of hierarchical structures on reality may be explained based on the Miller's [29] finding about the limits of human information processing capacity. Our short-term memory holds around seven units of information. In order to cope with a large amount of information, the chunking principle is applied so that individual pieces of information are chunked together to form one larger unit. One may successively obtain a sequence of chunks so that the number of units in each level is within the capacity of the short-term memory. This process of chunking leads naturally to a hierarchical structure. Our hierarchical thinking is determined by our limited capacity to process information. It is not surprising that hierarchical structures are used universally. For example, hierarchical structures are used in our study of languages and knowledge, as well as in our reading and writing $[15,31,60])$. It have also been argued by many authors that human beings consistently search for order and human inclination to assert order may lead to the conception of hierarchy [36, 62].

Both the objective and the subjective natures of levels are well discussed in systems science, where hierarchy is a central concept [1, 46, 49]. On the one hand, it is assumed that "[t]he Universe is a hierarchy of systems; that is, simple systems are synthesized into more complex systems from subatomic particles to civilizations" [49]. The concept of hierarchy is a universal principle existing in natural, conceptual and man-made systems. Real world complex systems tend to organize hierarchically. On the other hand, it is also recognized that the word system "does not refer to existing things in the real world but rather to a way of organizing our thought about the real world" [49]. The study of systems is based the integration of the two views.

\subsection{Separation and integration of levels}

Studies on levels normally consider two related issues, namely, separation and integration. According to Novikoff [35], "The concept of integrative levels recognizes as equally essential for the purpose of scientific analysis both the isolation of parts of a whole and their integration into the structure of the whole." The two aspects are interwoven together in a hierarchy. 
The separation of levels relies on two fundamental notions: loose coupling of parts in nearly-decomposable systems [47] and approximate knowledge [6]. On the one hand, it is important to realize that reality is a web in which everything is connected to everything else [6], and nature does not provide a picture where each level is clearly separated from the others. On the other hand, it is equally important to note that some things are more connected than others. We can explore the property of loose coupling in so-called nearly-decomposable systems to form various levels. Since such a separation of levels usually ignores subtle and small differences between individuals and their weak connections to others, the resulting multilevel hierarchical structures are approximations of reality. The knowledge obtained is in turn approximate. Nevertheless, such approximate knowledge is accurate and good enough for many practical purposes. The separation of levels thus gains in simplicity and clarity at the expense of accuracy.

Integration of levels is based on their interdependency and granularity. A level does not exist without its higher and/or lower levels. An ordering of levels is usually defined by the granularity of these levels. That is, different levels in general represent levels of differing complexity. Although a higher level depends on its lower levels, it has its unique properties that cannot be induced from lower levels. With integrative levels, we can easily shift our attention between different levels. By focusing on a particular level, we may study a specific aspect of reality.

Levels and hierarchies are the results of both separation and integration. Without separation, it is impossible to have levels; without integration, hierarchies do not exist. Levels are separated so that we can concentrate on a particular level at a specific point of time; levels are integrated so that we can observe the interworking of all levels in a hierarchy. In a hierarchy, we can study the interaction of levels. A hierarchy allows both analytical thinking through separation and synthetical thinking through integration. Separation and integration may therefore be viewed as two sides of the same coin.

The separation and integration of levels offer two methods for constructing and interpreting a hierarchy: the top-down methods and the bottom-up methods. The bottom-up methods may be explained based an evolutionary framework of systems, from lower levels to higher levels. In the context of biology, Novikoff [35] suggests that new a level of complexity emerges from lower levels through organization and integration of units. The wholes on a lower level become parts on a higher level. Conger [8] discusses three issues in the development of later levels from earlier levels, namely, "(1) integration, or creative synthesis, (2) combining relations, or mutuality of relations, and (3) emergence of new qualities." The topdown approaches offer a good choice for representation, description, and understanding. Hierarchical thinking appears to be natural to many of us. When a system or phenomenon is explained from a skeleton to details, or from using general terms to using specific terms, it is much easier for us to understand. An understanding on one level makes an understanding on another level feasible.

A good example involving both bottom-up and top-down approaches is the writing process given by Flower and Hayes $[12,13]$. In the phase of idea genera- 
tion, one may build the thesis of an article through a bottom-up approach, where scattered points, facts, and ideas are progressively synthesized into a whole. In writing up the article, a top-down approach is used, where the thesis is broken into parts and these parts are further broken into sub-parts, based on a scheme represented by an ideas tree. An article is a product with multiple levels of detail, consisting of the title, the headings of the sections and subsections, paragraphs and individual sentences [60]. Similarly, the reading process of such constructed articles also involves a multiple level understanding [15]. The integrative understanding may be explained by hermeneutic circle, namely, "our understanding of the parts hinges on our understanding of a larger whole, which, again, can only be understood on the basis of the parts" [44].

\section{A Short Survey on Studies of Levels}

In this section, we briefly review studies of levels. It is not our intent to provide a complete survey, but a set of examples that are pertinent to our understanding and formulation of granular computing. In fact, from Table 1 we can easily see that a complete survey on all uses of levels is almost an impossible task.

\subsection{Hierarchies in systems theory}

In his book, General Systems Theory, Ideas and Applications, Skyttner [49] reviews more than a dozen systems theories and points out that all of them share a set of common properties. All of them (except one) are formulated based on hierarchies of both complexity and size. In addition, such hierarchical structures exist at all levels and on all scales. The theory of hierarchy is central to the general systems theory and some authors refer the former as a dialect of the latter [2].

Detailed descriptions of various levels in each of the systems theories are given in the Skyttner's book [49]. The properties or laws of levels have been studied and stated by many authors $[2,11,35,41,42,51,52]$. The following list gives a few of them:

1. Levels are populated by entities whose properties and interaction determines the level in question. Levels are formed based on laws of nature on the one hand and based on our cognitive understanding of reality on the other hand.

2. Levels represent both a separation of wholes into parts and an integration of parts into wholes. Each level is relatively autonomous and complete. It is possible to study each level within itself, as well as in the context of other levels. All levels are also integrated; a disturbance introduced at any one level reverberates at all other levels. 
3. Levels are both continuous and discrete. New levels can always emerges from older levels in a continuous evolution and there may not exist a clear line that separates one level from another. At the same time, it is possible to identify various individual levels as the focal points of discussion.

4. Levels are ordered partially based on their corresponding complexity. At any given level, its mechanism lies at the level below and its purpose at the level above. A higher level normally has a smaller population of instances.

5. Each level depends on, and organizes, the level or levels below it. Each level has its emergent properties that cannot be deduced from lower levels. A level governs and controls its lower levels.

6. The knowledge of the lower level is necessary for a full understanding of the higher level; and yet it is impossible to predict the behavior of the high level based on such knowledge.

This is not a complete list and more properties may found in the given references. These properties are most relevant to our discussion on granular computing.

\subsection{Levels in cognitive science and psychology}

The concept of levels has been considered in cognitive science and psychology in many different forms. We focus mainly on the human acquisition, processing and utilization of knowledge at multiple levels.

In characterizing human knowledge, one needs to consider two topics, namely, context and hierarchy $[40,48]$. Knowledge is contextual and hierarchical. A context in which concepts are formed provides meaningful interpretation of the concepts. Knowledge is organized in a tower or a partial ordering. The baselevel, or first-level, concepts are the most fundamental concepts, and higher-level concepts depend on lower-level concepts. Level thinking is of fundamental importance in the understanding, representation, organization and synthesis of data, information, and knowledge. Such a structured organization of knowledge seems to be one way to get around the limited capacity of human information processing, which was shown by Miller [29] and discussed earlier.

Levels of processing theory, proposed by Craik and Lockhart [10], presents a model of human memory and information processing in memory. The theory is formulated on the basis that human "perception involves the rapid analysis of stimuli at a number of levels or stages." While the earlier stages, i.e., shallow levels, process physical or sensory features, the later stages, i.e., deep levels, are more concerned with pattern recognition and meaning extraction. This model of a hierarchy of processing stages reflects multiple levels of the depth of processing, where a greater depth implies a greater degree of semantic or cognitive analysis. A more plausible alternative to such a sequential progression, from shallow to deep, is a combination of both stimulus-driven bottom-up processing and concep- 
tually driven top-down processing [9]. The latter processing pattern seems to be consistent with the properties of levels discussed earlier.

Hierarchically structured knowledge has also been extensively explored in learning and student instruction. In teaching problem solving in physics, Reif and Heller [45] state, "effective problem solving in a realistic domain depends crucially on the content and structure of the knowledge about the particular domain." Knowledge about physics in fact specifies concepts and relations between them at various levels of abstraction. Furthermore, the knowledge is organized hierarchically, with explicit guidelines specifying when and how this knowledge is to be applied. Effective instruction needs to make effective use of such hierarchically structured knowledge. Posner [43] suggests that, according to the cognitive science approach, to learn a new field is to build appropriate cognitive structures and to learn to perform computations that will transform what is known into what is not yet known.

Levels of organization, levels of representation, levels of processing and levels of understanding are some of the important notions relevant to the study of human learning, information processing, and problem solving. The notion of levels helps us to explain and articulate many human activities and behaviors.

\subsection{Levels in computer science}

The notion of levels is widely used in computer science to describe and study various computer systems and concepts. With the introduction of levels, many concepts in computer science become easier to explain and understand. A few examples are discussed in this section.

In his work on vision, Marr [28] argues that a full understanding of an information processing system involves explanations at various levels. He proposes a three-level framework with each level addresses a particular of issues, which is quoted here:

1. Computational theory: What is the goal of the computation, why is it appropriate, and what is the logic of the strategy by which it can be carried out?

2. Representation and algorithm: How can this computational theory be implemented? In particular, what is the representation for the input and output, and what is the algorithm for the transformation?

3. Hardware implementation: How can the representation and algorithm be realized physically?

It can be said that each level addresses a different type of question, from abstract to concrete. The three levels are both dependent and independent.

Two basic notions, representation and process, are used to explain the threelevel framework. The representation deals with the explicit forms of entities or 
types of information, the process deals with the operations on entities. The most abstract level deals with what the process does and why. One builds a theory that explains internal working principles of the process, and defines the operations by specifying constraints that must be satisfied by the process. The second level deals with the realization of the process in an abstract way. One needs to choose a representation for the input and for the expected output of the process, and to specify an algorithm for the transformation from input to output. The choices of representation and algorithm are closely tied together. There usually exist many alternative representations. For a given representation, there are also many possible algorithms. A representation and an algorithm should be chosen so that advantages of the representation are fully exploited by the algorithm and, at the same time, the disadvantages of the representation are avoided. The third level deals with the physical realization of the process. The devices that physically realize a process may not be unique. The advances in technologies imply that a same process may be implemented again with the invention of new physical devices.

Investigations at the computational theory level are independent of representations, and investigations at the representation and algorithm level is independent of physical devices. The levels are ordered and interpreted as levels of abstraction. The representation and algorithm level can also be named as logical implementation level, and the hardware implementation level as the physical implementation level.

In developing a theory of the nature of knowledge and representation, Newell $[32,33]$ introduces the concept of knowledge level. He views the nature of knowledge as the medium of a system level that lies above the symbol or program level. This is summarized by the so-called Knowledge Level Hypothesis [32]: "There exists a distinct computer systems level, lying immediately above the symbol level, which is characterized by knowledge as the medium and the principle of rationality as the law of behavior." A framework of computer system levels thus consists of the device level, the circuit level, the logic level (with its two sublevels, combinatorial and sequential circuits, and the register-transfer level), the program or symbolic level, the knowledge level, and the configuration level (which is supported by the preceding three levels).

The systems levels as defined above are precise and operational. A level consists of a medium, components, laws of composition, and laws of behavior. Each level processes its medium based on the primitive processing provided by components, laws for assembling the components to form systems, and laws for determining the system behavior. There are additional characteristics of system levels. A given level may be implemented in many variant ways. Each level can be defined autonomously without reference to other levels, and each level can be reduced to, or realized by, the level below. Specification of a system at a particular level determines the system behavior at that level. The behavior of the total system is a combined result of local effects of all components. It is interesting to note some of these properties have also been extensively studied in systems science. 
In the context of algorithm design, Foster [14] critically reviews and systematically compares various definitions and interpretations of the notion of levels. Three basic issues, namely, definition of levels, number of levels, and relationship between levels, are clarified. Three important points made by Foster are summarized as follows. First, levels are considered simply as descriptions or points of views and often for the purpose of explanation. Second, the number of levels is not fixed, but depends on the context and the purpose of description or explanation. Third, levels can be graphically represented as a vertical stack of planes. This multi-layered theory of levels captures two senses of abstraction. One is the abstraction in terms of concreteness and is represented by planes along the dimension from top to bottom. The other is the abstraction in terms of the amount of detail and can be modeled along another dimension from less detail to more detail on the same plane. The two senses of abstraction can be interpreted in terms of multiple hierarchies. The main hierarchy consists of levels, and each level is a hierarchy consisting of different levels of detail. For example, in the Marr's three-level hierarchy, the logical implementation level may be a hierarchy consisting of logical implementations in various details. The abstraction in terms of detail is very useful in the implementation of information processing systems.

An excellent example of the effective use of levels is structured programming, characterized by top-down design and stepwise refinement. There is a huge body of literature on this topic. The following steps, taken from Ledgard et al. [25], are perhaps sufficient to illustrate the basic ideas:

1. Design in levels: A level consists of a set of modules. At higher levels, only a brief description of a module is provided. The details of the module are to be refined, divided into smaller modules, and developed in lower levels.

2. Initial language independence: The high-level representations at initial levels focus on expressions that are relevant to the problem solution, without explicit reference to machine and language dependent features.

3. Postponement of details to lower levels: The initial levels concern critical broad issues and the structure of the problem solution. The details such as the choice of specific algorithms and data structures are postponed to lower, implementation levels.

4. Formalization of each level: Before proceeding to a lower level, one needs to obtain a formal and precise description of the current level. This will ensure a full understanding of the structure of the current sketched solution.

5. Verification of each level: The sketched solution at each level must be verified, so that errors pertinent to the current level will be detected.

6. Successive refinements: Top-down programming is a successive refinement process. Starting from the top level, each level is redefined, formalized, and verified until one obtains a full program.

It is an easy task to apply the same principles elsewhere. For example, it has been suggested that the top-down approach is effective for developing, communicating 
and writing mathematical proofs $[16,23,26]$. The same principles can be applied in the preparation, organization, and writing of scientific articles $[13,60]$.

The operational feature of the notion of levels in computer science perhaps needs more emphasis. While the notion of levels is used in some disciplines as a tool for explanation, it is fully implemented in computer science in systems design, programming and many more. These actual implementations may provide necessary hints for implementing levels in related disciplines.

\section{Implications of Integrative Levels to Granular Computing}

The recent rise of granular computing may be compared with the rise of systems theory a few decades earlier, in terms of their philosophies, goals, scopes, and methodology [61]. The general systems theory attempts to discover and investigate structures and underlying principles common to most of natural and artificial systems $[6,17,24,49,51])$. The general systems theory is viewed as a science of sciences $[49,51]$ in an attempt to arrive at unity through diversity [17]. Similarly, research of granular computing attempts to discover and investigate structures and underlying principles common to most types of human problem solving [61]. As such, granular computing may be viewed as human-inspired computing and problem solving.

An important feature of human intelligence is that humans have many "Ways to Think" and can also create new "Ways to Think", as suggested by Minsky [30]. Another feature is that humans form multiple representations of the world. This, in another way, motivates the study of granular computing. Granular computing can be viewed as a particular class of such "Ways to Think" that focuses on multiple levels of granularity. Furthermore, the notion of integrative levels may well serve the purpose of multiple representations.

Given this interpretation of granular computing, we can examine several important implications of the notion of integrative levels.

It is evident that the concept of integrative levels is very essential to human problem solving and has been used effectively time and again in many different disciplines. There seems to be a common set of interpretations, heuristics, principles and strategies of problem solving that are based on integrative levels. Unfortunately, these principles are scattered over many places in isolation without being synthesized into an integrated whole. They are normally explained with reference to discipline-specific knowledge and thus are buried deeply in minute details. Typically, the same principles are discussed in different languages and notations. In many occasions, we use these principles either implicitly or subconsciously because a formal documentation does not exist. This has led to the reinvention of the same principles time and again in the same or different fields.

The systems theory, to some extent, has resolved some of these problems. By introducing granular computing as a new field of study, we focus on a particular 
aspect. The notion of granularity is introduced to interpret the concept of integrative levels, and thus we have the notion of integrative levels of granularity. Each level is populated with granules of similar size or of similar nature. The levels of granularity may be interpreted as the levels of organization, levels of control, levels of complexity, levels of understanding, levels of description, levels of representation, levels of interpretation, levels of abstraction, levels of details, levels of processing and so on. The universality of levels implies that integrative levels of granularity may be used as a basis for granular computing.

The subjective view of levels suggests a hierarchy may only reflect our perception of reality and hence is only an approximation of reality. To remedy the shortcomings of such an approximation, it is necessary to consider many hierarchies in order to obtain multiple views of the same world $[7,30,58]$. With integrative levels of granularity, we consider granular structures that represent both multiple levels and multiple views. A single hierarchy gives one multilevel view of reality; many hierarchies give a mulitview description of reality [58]. Granular computing explores both of them.

The separation and integration of levels, together with the associated bottom-up and top-down methods, are related to the methodology of granular computing. The properties and laws of levels are useful in constructing various levels when applying granular computing principles in different domains. The implementation of levels in computer science offers more concrete ideas for applying ideas of granular computing.

\section{The Triarchic Theory of Granular Computing}

Once we accepted the notion of integrative levels of granularity as a basic concept of granular computing, we in fact emphasize a research direction that is dominated by structuredness. The study of granular computing depends crucially on granular structures that represent reality through multilevel and multiview.

The triarchic theory is a unified view that stresses the study of granular computing as a new field in its wholeness, rather than scattered pieces. Based on multiple level hierarchical structures, the triarachic theory integrates philosophical, methodological, and computational issues of granular computing as structured thinking, structured problem solving and structured information processing, respectively. A brief description of the theory is given in this section and more details can be found in $[57-59,61]$.

The core of the triarchic theory can be pictorially described by the granular computing triangle. The three vertices of the triangle represent the philosophical, methodological and computational perspectives.

Philosophy: granular computing as structured thinking. The philosophy of granular computing offers a worldview characterized by different sized, interact- 
ing and hierarchically organized granules $[19,38,63,64]$. This view of the world in terms of structures, as represented by multiple integrative levels, leads to a way of structured thinking, which is applicable to many branches of natural and social sciences. Broadly speaking, granular computing draws results from two complementary philosophical views about the complexity of real-world problems, namely, the traditional reductionist thinking and the more recent systems thinking. It combines analytical thinking for decomposing a whole into parts and synthetic thinking for integrating parts into a whole.

Methodology: granular computing as a general method of structured problem solving. Granular computing promotes systematic approaches, effective principles, and practical heuristics and strategies that have been used effectively by humans for solving real-world problems. A central issue is the exploration of granular structures. This involves three basic tasks: constructing granular structures, working within a particular level of the structure, and switching between levels. We can formulate a set of principles to highlight the methodology of granular computing. For example, the principle of multilevel granularity emphasizes the effective use of a hierarchical structure. According to this principle, we must consider multiple representations at different levels of granularity. The principle of multiview stresses the consideration of diversity in modeling. We need to look at the same problem from many angles and perspectives. Once granular structures are obtained, we can apply other principles to work based on such structures. For example, the principle of focused efforts calls for attention on the focal point at a particular stage of problem solving; the principle of granularity conversion links the different stages in this process. The principle of view switching allows us to change views and to compare different views. These principles of granular computing have, in fact, been used extensively in different disciplines under different names and notations. Many principles of structured programming can be readily adopted for granular computing.

Computation: granular computing as a new paradigm of structured information processing. Granular computing focuses on information processing methods based on the granular structures $[4,5]$. The term computing needs to be understood in its broad meaning to include information processing in the abstract, in the brain and in machines. While information processing in the abstract deals with theories of computing without direct reference to their implementations, information processing in the brain and in machines represents the biological (natural) and the physical (artificial) implementations, respectively. Two related basic issues of computation are representations and processes (operations). Representation covers the formal and precise description of granules and granular structures. Processes may be broadly divided into the two classes: granulation and computation with granules. Granulation processes involve the construction of the building blocks and structures, namely, granules, levels, and hierarchies. Computation 
processes explore the granular structures. This involves two-way communication up and down in a hierarchy, as well as switching between levels.

The three perspectives of granular computing are connected and mutually support each other. A reviewer of this chapter points out the importance of studying the interactions of the three perspectives. The reviewer states, "In particular, a general picture illustrating interactions within the triangle would be helpful. ... Speaking more about possible scenarios of interactions may turn out to be even more valuable than speaking about particular 'nodes' of the triangle." In some sense, the three perspectives can be interpreted as three levels of study, with the philosophical foundations supporting the methodological foundations, which in turn supports the computational implementations [57, 58]. It immediately follows that the arguments of separation and integration of levels can be directly applied to the separation and integration of three perspectives on granular computing.

With the separation of three perspectives, we emphasize the importance of philosophical and methodological studies that have played a relatively minor role so far. Granular computing offers a new way of thinking that may lead to a new set of problem-solving methods, or more precisely, a recasting of many existing methods in a new setting. Unfortunately, granular ways of thinking are not fully appreciated yet, due to some computational issues. Doubts on the potential of granular computing are commonly expressed at the computational level, as there still does exist a set of well-accepted algorithms or computational methods for granular computing. In [61], we argue that a lack of recent progresses in artificial intelligence may perhaps be explained by a viewpoint that paid little attention to human intelligence and how the brain works. A new school of thought is emerging that emphasizes the study of human brains and natural intelligence. If we view granular computing as human-inspired computing, we must study how humans solve problems by exploiting multiple levels of granularity. Consequently, we need to pay attention to the philosophical and methodological foundations of granular computing.

There is a top-down guiding role played by the three levels of granular computing. The philosophy of granular computing will guide us in searching for the right methodology; the methodology in turn can be applied in the design and implementation of granular-computing-based information systems. The separation of the three perspectives enables to us to ask the right questions and choose the right languages for granular computing at three levels. The philosophy of granular computing can be described in general terms. Its applications lead to two related classes of methodology, one for human problem solving and the other for machine problem solving. The former is more general than the latter; the latter is a specialization of the former. While methodology for humans may be qualitative and schematic, the methodology for machines must be precise and formal. At the next level, the methodology of granular computing is applied to concrete implementations. There is also a reverse bottom-up way of support. A study of granular- 
computing-based systems may offer new methodology, which in turn may help us in redefining our philosophical standing.

The three-level interpretation of granular computing is convenient, but of limited value. In general, the three perspectives are on the same footing and mutually support each other. That is, one node of the triangle supports, and is supported by, the other two; one cannot exist without the other two. This requires an integrated view that granular computing has three indispensable components. Any study that focuses only on some aspects may fail to realize the full potential of granular computing.

The triarchic theory puts granular computing research on a firm basis. In addition, the granular computing triangle recommends a research direction towards an interdisciplinary wholeness approach. That is, researchers in different disciplines may investigate different perspectives of granular computing and at the same time integrate their individual results.

\section{Conclusion}

The chapter examines a central notion of granular computing, namely, integrative levels of granularity. Two main features of integrative levels are discussed: the objective and subjective views of levels, and the separation and integration of levels. A survey on integrative levels and their basic properties, in several disciplines, suggests that integrative levels of granularity may serve as a basis for the study of granular computing. The triarchic theory of granular computing is briefly reviewed based on this notion.

Future progresses on the study of granular computing cannot be achieved based merely on investigations of concrete models or methods, namely, the computational perspective. Successful applications of granular computing may justify its existence; but they alone are far from enough. One needs to look at its foundations and roots [4]. In this regards, a conceptual framework, such as the triarchic theory, may be helpful.

\section{Acknowledgements}

The author is grateful to an anonymous reviewer and Dr. Andrzej Bargiela for their constructive comments. In particular, the author would like to thank the reviewer for pointing out the needs for studying interactions between three nodes of the granular computing triangle. 


\section{References}

1. Ahl, V. and Allen, T.F.H. (1996) Hierarchy Theory, a Vision, Vocabulary and Epistemology, Columbia University Press.

2. Allen, T.F. A Summary of the Principles of Hierarchy Theory, http://www.isss.org/hierarchy.htm (accessed March 11, 2005).

3. Bargiela, A. and Pedrycz, W. (2002) Granular Computing: An Introduction, Boston: Kluwer Academic Publishers.

4. Bargiela, A. and Pedrycz, W. (2006) The roots of granular computing, Proceedings of 2006 IEEE International Conference on Granular Computing, 806-809, 2006.

5. Bargiela, A. and Pedrycz, W. (2008) Toward a theory of granular computing for humancentered information processing, IEEE Transactions on Fuzzy Systems, to appear.

6. Capra, F. (1997) The Web of Life, New York: Anchor Books, 1997.

7. Chen, Y.H. and Yao, Y.Y. (2008) A multiview approach for intelligent data analysis based on data operators, Information Sciences, 178, 1-20.

8. Conger, G.P. (1925) The doctrine of levels, The Journal of Philosophy, 22, 309-321.

9. Craik, F.I.M. (2002) Levels of processing: past, present ... and future? Memory, 10, 305318.

10. Craik, F.I.M. and Lockhart, R.S. (1972) Levels of processing: a framework for memory research, Journal of Verbal Learning and Verbal Behavior, 11, 671-684.

11. Feibleman, J.K. (1954) Theory of integrative levels, The British Journal for the Philosophy of Science, 5, 59-66.

12. Flower, L. (1981) Problem-Solving Strategies for Writing, New York: Harcourt Brace Jovabovich, Inc.

13. Flower, L.S. and Hayes, J.R. (1977) Problem-solving strategies and the writing process, College English, 39, 449-461.

14. Foster, C.L. (1992) Algorithms, Abstraction and Implementation: Levels of Detail in Cognitive Science, London: Academic Press.

15. Frank, S.D. (1990) Remember Everything You Read, New York: Times Books.

16. Friske, M. (1995) Teaching proofs: a lesson from software engineering, American Mathematical Monthly, 92, 142-144.

17. Gray, W. and Rizzo, N D. (Eds.) (1973) Unity Through Diversity, I \& II, New York: Gordon and Breach Science Publishers.

18. Hawkins, J. (with Blakeslee, S.) (2004) On Intelligence, New York: Henry Holt and Company.

19. Hobbs, J.R. (1985) Granularity, Proceedings of the Ninth International Joint Conference on Artificial Intelligence, 432-435.

20. Inuiguchi, M., Hirano, S. and Tsumoto S. (Eds.) (2003) Rough Set Theory and Granular Computing, Berlin: Springer.

21. Keet, C.M. (2006) A taxonomy of types of granularity, Proceeding of 2006 IEEE International Conference on Granular Computing, 106-111.

22. Keet, C.M. (2008) A Formal Theory of Granularity, PhD Thesis, KRDB Research Centre, Faculty of Computer Science, Free University of Bozen-Bolzano, Italy, http://www.meteck.org/files/AFormalTheoryOfGranularity_CMK08.pdf (accessed June 8, 2008).

23. Lamport, L. (1995) How to write a proof, American Mathematical Monthly, 102, 600-608.

24. Laszlo, E. (1972) The Systems View of the World: the Natural Philosophy of the New Developments in the Science, New York: George Brasiller.

25. Ledgard, H.F., Gueras, J.F. and Nagin, P.A. (1979) PASCAL with Style: Programming Proverbs, Rechelle Park, New Jersey: Hayden Book Company, Inc.

26. Leron, U. (1983) Structuring mathematical proofs, American Mathematical Monthly, 90, 174-185. 
27. Lin, T.Y., Yao, Y.Y. and Zadeh, L.A. (Eds.) (2002) Data Mining, Rough Sets and Granular Computing, Heidelberg: Physica-Verlag.

28. Marr, D. (1982) Vision, A Computational Investigation into Human Representation and Processing of Visual Information, San Francisco: W.H. Freeman and Company.

29. Miller, G.A. (1956) The magical number seven, plus or minus two: some limits on our capacity for processing information, Psychological Review, 63, 81-97.

30. Minsky, M. (2006) The Emotion Machine: Commonsense Thinking, Artificial Intelligence, and the Future of the Human mind, New York: Simon \& Schuster Paperbacks.

31. Minto, B. (2002) The Pyramid Principle: Logic in Writing and Thinking, London: Prentice Hall/Financial Times.

32. Newell, A. (1982) The knowledge level, Artificial Intelligence, 18, 87-127.

33. Newell, A. (1993) Reflections on the knowledge level, Artificial Intelligence, 59, 31-38.

34. Nguyen, S.H., Skowron, A. and Stepaniuk, J. (2001) Granular computing: a rough set approach, Computational Intelligence, 17, 514-544.

35. Novikoff, A.B. (1945) The concept of integrative levels and biology, Science, 101, 209 215.

36. Patrides, C.A. (1973) Hierarchy and order, in: Dictionary of the History of Ideas, Vol. 2, New York: Scribners, 434-449

37. Pattee, H.H. (1973) Unsolved problems and potential applications of hierarchy theory, in: Hierarchy Theory, The Challenge of Complex Systems, Pattee, H.H. (Ed.), New York: George Braziller, 129-156.

38. Pawlak, Z. (1998) Granularity of knowledge, indiscernibility and rough sets, Proceedings of 1998 IEEE International Conference on Fuzzy Systems, 106-110.

39. Pedrycz, W., Skowron, A. and Kreinovich, V. (Eds.) (2008), Handbook of Granular Computing, New York: Wiley.

40. Peikoff, L. (1991) Objectivism: the Philosophy of Ayn Rand, New York: Dutton

41. Poli, R. (1998) Levels, Axiomathes, 9, 197-211.

42. Poli, R. (2002) The basic problem of the theory of level of reality, Axiomathes, 12, 261 283.

43. Posner, M.I. (Ed.) (1989) Foundations of Cognitive Science, Cambridge, Massachusetts: The MIT Press.

44. Ramberg, B. and Gjesdal, K. (2005) Hermeneutics, The Stanford Encyclopedia of Philosophy, http://plato.stanford.edu/entries/hermeneutics/ (accessed June 14, 2008).

45. Reif, F. and Heller, J. (1982) Knowledge structure and problem solving in physics, Educational Psychologist, 17, 102-127.

46. Salthe, S.N. (1985) Evolving Hierarchical Systems, Their Structure and Representation, Columbia University Press.

47. Simon, H.A. (1973) The organization of complex systems, in: Hierarchy Theory, The Challenge of Complex Systems, Pattee, H.H. (Ed.), New York: George Braziller, 1-27.

48. Simpson, S.G. (1996) What is foundations of mathematics? http://www.math.psu.edu/simpson/hierarchy.html (accessed November 21, 2003).

49. Skyttner, L. (2001) General Systems Theory, Ideas \& Applications, Singapore, World Scientific.

50. Verdier, B. (2006) Hierarchy: a short history of a word in western thought, in: Hierarchy in Natural and Social Sciences, Pumain, D. (Ed.), The Netherlands: Springer, 13-37.

51. von Bertalanffy, L. (1950) An outline of general system theory, The British Journal for the Philosophy of Science, 1, 134-165.

52. Wilson, T.D. (2003) Philosophical foundations and research relevance: issues for information research, Journal of Information Science, 29, 445-452.

53. Yao, J.T. (2007) A ten-year review of granular computing, Proceedings of 2007 IEEE International Conference on Granular Computing, 734-739.

54. Yao, Y.Y. (2000) Granular computing: basic issues and possible solution, Proceedings of the 5th Joint Conference on Information Sciences, 186-189. 
55. Yao, Y.Y. (2004a) A partition model of granular computing, LNCS Transactions on Rough Sets I, LNCS 3100, 232-253.

56. Yao, Y.Y. (2004b) Granular computing, Computer Science (Ji Suan Ji Ke Xue), 31,1-5.

57. Yao, Y.Y. (2005) Perspectives of granular computing, Proceedings of 2005 IEEE International Conference on Granular Computing, 85-90.

58. Yao, Y.Y. (2006) Three perspectives of granular computing, Journal of Nanchang Institute of Technology, 25, 16-21

59. Yao, Y.Y. (2007a) The art of granular computing, Proceeding of the International Conference on Rough Sets and Emerging Intelligent Systems Paradigms, LNAI 4585, 101-112.

60. Yao, Y.Y. (2007b) Structured writing with granular computing strategies, Proceedings of 2007 IEEE International Conference on Granular Computing, 72-77.

61. Yao, Y.Y. (2008) The rise of granular computing, Journal of Chongqing University of Posts and Telecommunications (Natural Science Edition), 20, 299-308.

62. Young, G.L. (1978) Hierarchy and central place: some questions of more general theory, Geografiska Annaler, Series B, Human Geography, 60, 71-78.

63. Zadeh, L.A. (1997) Towards a theory of fuzzy information granulation and its centrality in human reasoning and fuzzy logic, Fuzzy Sets and Systems, 90, 111-127.

64. Zhang, L. and Zhang, B. (2007) Theory and Application of Problem Solving - Theory and Application of Granular Computing in Quotient Spaces, 2nd edition, Beijing: Tsinghua University Press. 